\title{
Uma Comparação dos Dados da Ferramenta Zabbix com a Percepção dos Usuários da Rede no Instituto Federal Catarinense - Campus Avançado Sombrio
}

\author{
Diuliana de Matos da Rosa ${ }^{1}$, Liliana Campos Colares ${ }^{1}$, Matheus Lorenzato Braga ${ }^{1}$, \\ Victor Martins de Sousa ${ }^{1}$ \\ ${ }^{1}$ Instituto Federal Catarinense - Campus Avançado Sombrio (IFC-CAS) \\ Sombrio - SC - Brasil \\ \{diulymatos, colares.liliana\} egmail.com, \\ \{matheus.braga, victor.sousa\} @ifc.edu.br
}

\begin{abstract}
This paper presents an analysis of users' memory in relation to the computer network of the Instituto Federal Catarinense - Campus Avançado Sombrio. The main questions were to raise the time of day with greater data flow, to verify if there is slow use of the network and at what times users perceive this problem. The survey was based on a questionnaire applied to users, comparing the answers and the graphs of the Zabbix monitoring tool. The analyzed users study at night shift and through the comparison it was possible to realize that there is a high rate of Internet access at night.
\end{abstract}

Resumo. Este trabalho apresenta uma análise da percepção dos usuários em relação à rede de computadores do Instituto Federal Catarinense - Campus Avançado Sombrio. As principais questões abordadas foram com o intuito de levantar o período do dia com maior fluxo de dados, verificar se existe lentidão no uso da rede e em quais horários os usuários percebem este problema. A pesquisa se deu com base em um questionário aplicado aos usuários, realizando uma comparação entre as respostas e os gráficos da ferramenta de monitoramento Zabbix. Os usuários analisados estudam no turno da noite e através do comparativo foi possível perceber que existe um alto índice de acesso à Internet no período noturno.

\section{Introdução}

Desde o seu surgimento, as redes de computadores têm crescido fortemente e vem se tornando essencial para a comunicação, troca de informações e compartilhamento de recursos. Ao mesmo tempo em que as redes oferecem várias oportunidades para a comunicação e compartilhamento de informações, criou-se um desafio em relação ao seu gerenciamento (LEONHARDT, 2005).

As redes de computadores, de acordo com Franciscatto, Cristo e Berlin (2014), podem ser definida como um conjunto de dois ou mais computadores que são interligados e possuem o objetivo de fazer o compartilhamento de recursos e trocar informações. Além disto, as redes estão cada vez mais presentes no dia a dia e englobando cada vez mais pessoas.

Um exemplo onde as redes de computadores estão presentes são em instituições de ensino e em grandes empresas, no qual é extremamente importante obtê-las pelo fato de que é necessário realizar pesquisas, trabalhos, trocar informações e fazer o compartilhamento de recursos entre os computadores, tornando o estudo e trabalho mais 
eficiente. Entretanto, não basta apenas ter uma boa rede, e sim é necessário monitorá-la para que a mesma apresente bons resultados aos seus usuários.

O gerenciamento de redes é essencial para que as redes funcionem corretamente com um bom nível de desempenho. Para que isso ocorra, é necessário a utilização de ferramentas específicas para efetuar estes gerenciamentos. Mesmo as redes menores e mais simples possuem essa necessidade. Além disso, com o gerenciamento é possível garantir aos seus usuários uma disponibilidade de serviços com um desempenho satisfatório. Franceschi (1999) salienta que sistemas de redes são difíceis de operar, diagnosticar e detectar problemas. Para isso, existe a necessidade de possuir uma gerência que controle a rede, aumentando sua eficiência e assegurando a produtividade.

O Instituto Federal Catarinense - Campus Avançado Sombrio (IFC-CAS) é um exemplo de redes de computadores presentes em instituições de ensino. Os usuários da rede utilizam diariamente um sistema acadêmico, facilitando o acesso em materiais de aula, informações institucionais, entre outros. Além da Internet disponibilizada, o IFCCAS possui computadores na biblioteca, nos laboratórios de informática e também nos setores administrativos. Sendo assim, possui um alto fluxo de informação pela rede, o que pode resultar em uma lentidão ou gargalo em determinados pontos ou períodos do dia, de acordo com reclamações de usuários da instituição.

Para que esta rede tenha um gerenciamento adequado os técnicos do setor de Tecnologia da Informação (TI) utilizam ferramentas específicas que os auxiliam para esta tarefa. Atualmente a ferramenta utilizada é o Zabbix. Conforme Black (2008), o Zabbix é uma das ferramentas que possui muitas opções de gerenciamento e é um software que, até o presente momento, está recebendo atualizações de software frequentes, com a capacidade de cobrir lacunas deixadas por seus concorrentes.

Além disso, no trabalho de Gomes (2018), o qual apresenta o monitoramento em uma instituição de ensino, conclui que a "implementação do Zabbix na rede acadêmica consegue trazer grandes benefícios, pois ele traz um melhor controle dos ativos na rede e consegue monitorar com precisão o ambiente em geral”.

Desta forma, este trabalho tem como objetivo aplicar um questionário com os usuários da rede IFC-CAS e com isso realizar uma comparação com os gráficos gerados pela ferramenta utilizada para o monitoramento da rede, em diferentes períodos do dia.

\section{Ferramenta de Monitoramento Zabbix}

De acordo com o site oficial, o Zabbix é uma ferramenta que realiza o monitoramento, acompanhamento de desempenho de aplicações, análise de experiência de usuário e análise de causa raiz em ambientes complexos. É uma ferramenta open source que foi desenvolvida em 1998 e desde então continuou crescendo.

Segundo Lima (2014), o Zabbix tem a capacidade de monitorar muitos itens com apenas um servidor e é possível também obter um monitoramento distribuído. Outra característica importante é que esta ferramenta apresenta um sistema com relatórios e gráficos, facilitando a visualização e também o acesso em datas e horários, além de que é possível avaliar todos os dados em tempo real.

A configuração para a realização do monitoramento da rede é efetuada através de uma interface web e a ferramenta em si é bem acessível, podendo coletar dados por meio de alertas, ações, itens e comandos remotos.

Algumas características principais do Zabbix são: administração e 
monitoramento via interface web, descobrimento de servidores e dispositivos de redes de forma automática, flexibilidade, monitoramento agregado, monitoramento distribuído, monitoramento em tempo real, sistemas de notificação, autenticação segura de usuários, visualização de relatórios, gráficos e mapas, entre outros (LIMA, 2014).

\section{Ambiente de Pesquisa}

A pesquisa deste trabalho foi realizada no Instituto Federal Catarinense - Campus Avançado Sombrio, localizado no bairro Januária em Sombrio - Santa Catarina. Importante ressaltar que, este trabalho encontra-se em uma fase inicial de pesquisa; assim, os equipamentos existentes e a topologia de rede atual na instituição ainda não foram levantados.

Na atualidade, o IFC-CAS possui cursos de nível médio/técnico e ensino superior, sendo eles: Técnico em Informática Integrado ao Ensino Médio, Técnico em Hospedagem Integrado ao Ensino Médio, Gestão de Turismo, Licenciatura em Matemática e Tecnologia em Redes de Computadores. Desta maneira, a instituição possui aproximadamente 670 alunos ativos.

Portanto, o presente trabalho analisa a percepção dos usuários da rede do IFCCAS em relação ao desempenho da rede tanto cabeada quanto WiFi. Em relação ao link de Internet, pode-se dizer que a instituição possui um link de $100 \mathrm{mb}$ que deve atingir todos os setores da instituição como as salas de aula, laboratórios de informática, biblioteca, setores administrativos, entre outros.

\section{Procedimentos Metodológicos}

A elaboração do presente trabalho foi feita a partir de um questionário aplicado com os usuários da rede do IFC-CAS e também por meio da solicitação de gráficos da ferramenta de monitoramento que o instituto utiliza, o Zabbix. Segundo Horst, Pires e Déo (2015) esta ferramenta possui muitas funções, e algumas das principais são a autodescoberta de dispositivos de rede e de recursos do host, a criação de forma automática dos gráficos para diferentes recursos do host escolhido para ser monitorado e também realiza o monitoramento com a administração via web por meio do uso do proxy.

É importante saber os períodos do dia em que os usuários sentem que o desempenho da rede diminui, pois de acordo com as respostas obtidas pode-se sugerir melhorias. Para isso, o questionário aplicado foi efetuado com a ferramenta Google Forms e é baseado em perguntas referente a rede e se existe lentidões durante o uso. Além disso, também é feito o questionamento sobre os horários em que é possível perceber uma lentidão maior no desempenho da rede. Portanto, além destes dados também foram requisitados os gráficos da ferramenta Zabbix ao setor de TI. Eles mostram o monitoramento da rede e os picos de acesso de um dia específico ou até de uma semana inteira.

Portanto, no questionário realizado foram aplicadas as seguintes questões:

1 - "Você considera o sinal do WiFi presente em todos os ambientes da Instituição?”

2 - "Você percebe que a rede WiFi apresenta lentidões durante o uso?”

3 - "Caso afirmativo para a questão anterior, qual período percebe a lentidão? (Pode ser assinalada mais de uma alternativa)." 
O acesso ao questionário foi por meio de um link disponibilizado aos usuários da rede, que responderam as questões nos computadores dos laboratórios de informática da instituição. A partir dos dados obtidos, foi possível realizar uma comparação entre as respostas do público em relação a lentidão nos diferentes períodos do dia com o gráfico do Zabbix.

\section{Resultados}

Para a obtenção dos resultados neste trabalho, um questionário foi aplicado com os usuários da rede de computadores do IFC-CAS, além de gráficos que foram solicitados ao setor de Tecnologia da Informação (TI).

Em relação ao questionário, de acordo com a pergunta 1 realizada como respostas obteve-se 78,9\% "Sim”, 10,5\% “Não" e 10,5\% “Não se aplica”. Analisando essas respostas podemos perceber que, de um modo geral, o nível de sinal de WiFi nas dependências da instituição é bastante satisfatório, pois obteve um alto valor de resposta positiva. A questão 2 abordada em relação à rede, as respostas dividiram-se entre "Sim" e "Não" como mostra a Figura 1.

Figura 1: Você percebe que a rede WiFi apresenta lentidões durante o uso?

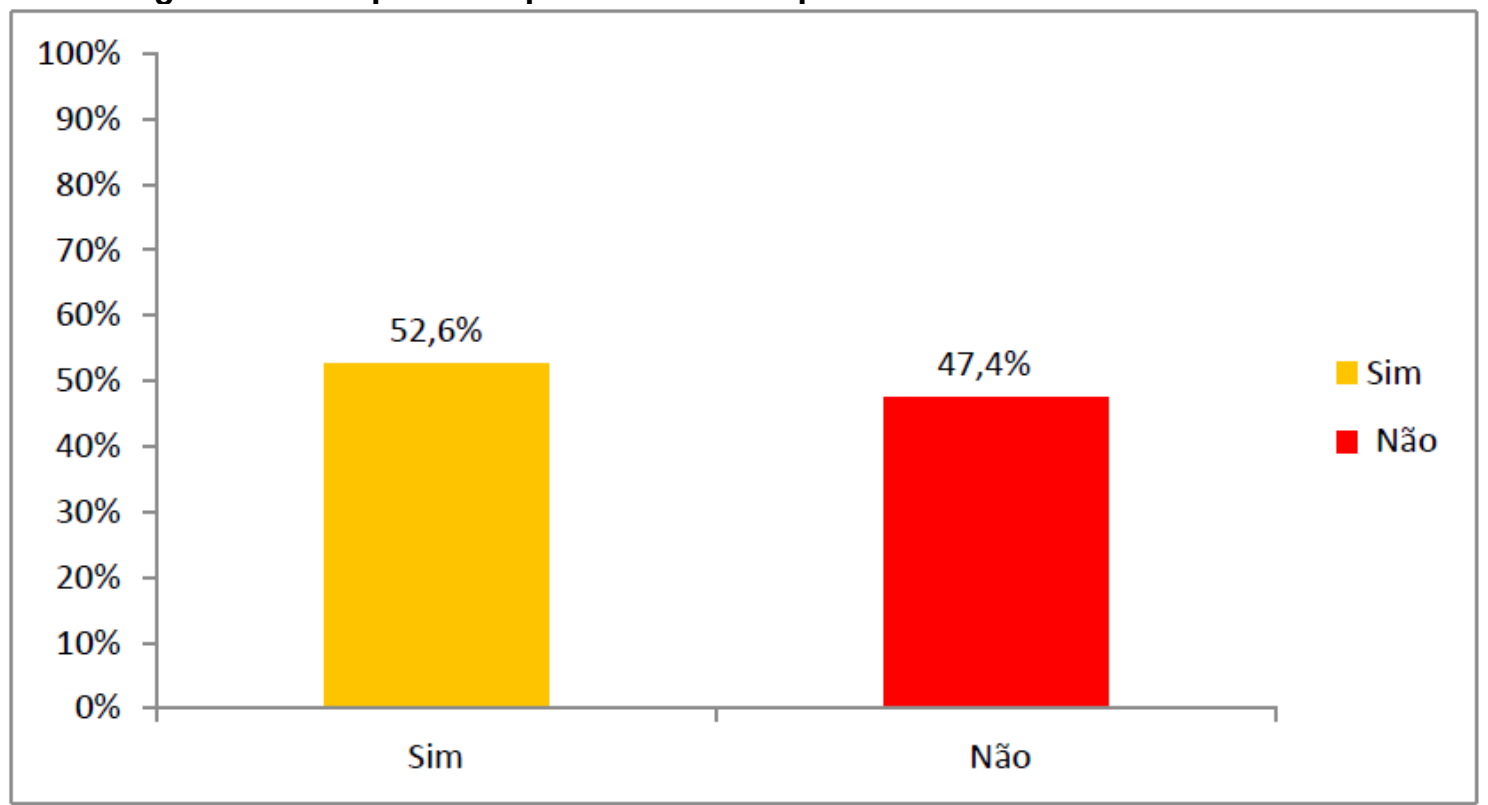

Fonte: Os autores, 2019.

Assim, podemos inferir que mais da metade dos usuários percebe algum período de lentidão no acesso à internet. Após, na pergunta 3 feita aos usuários sobre a lentidão da rede, como opções de respostas, haviam diferentes horários e o resultado é apresentado a seguir na Figura 3. 
Figura 2: Qual período percebe a lentidão? (Pode ser assinalada mais de uma alternativa).

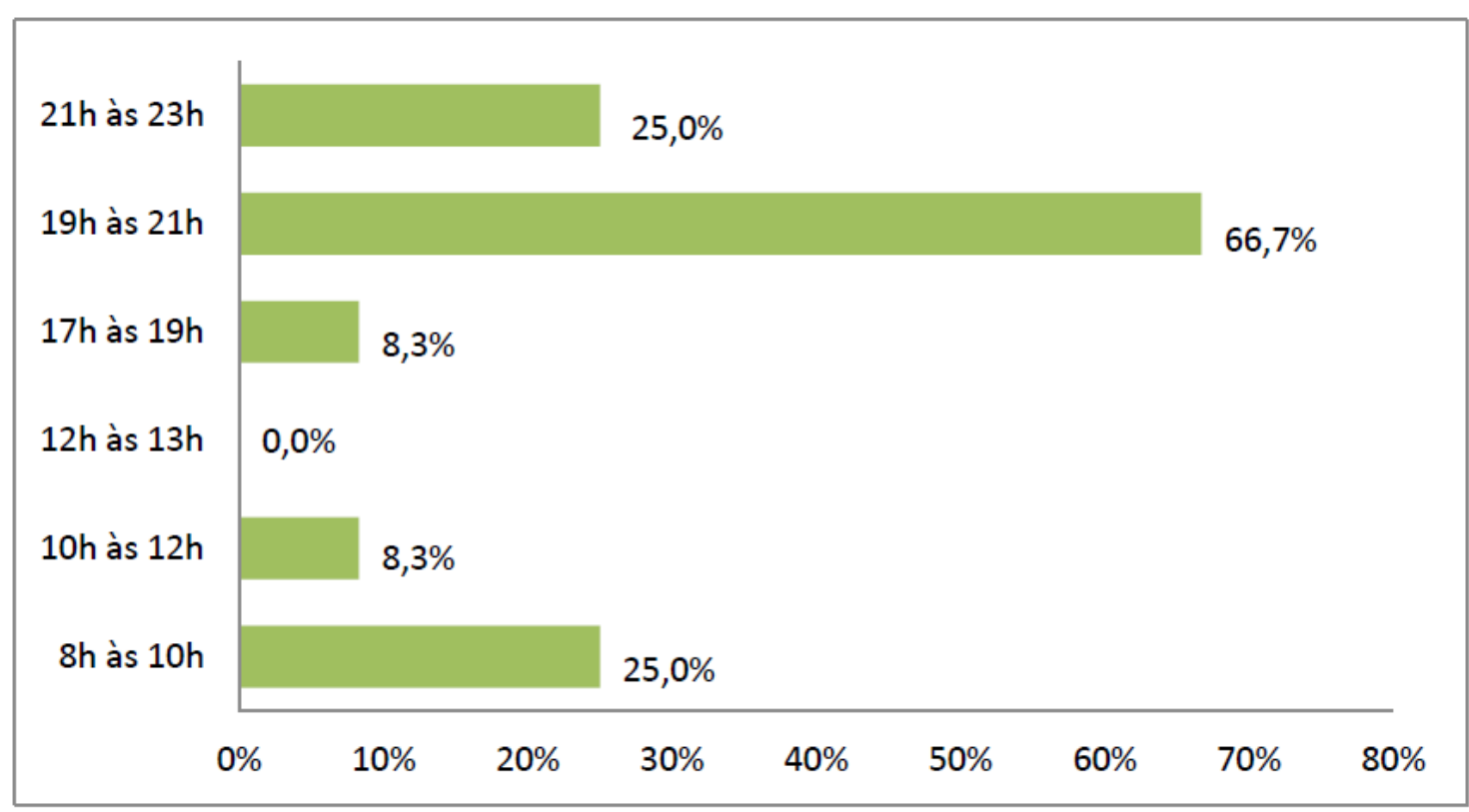

Fonte: Os autores, 2019.

Ao analisar a Figura 2, pode-se perceber que o período que apresenta maior lentidão é no início das aulas no período noturno (19 horas). Por se tratar de um trabalho em iniciação, os usuários analisados foram somente do turno da noite, sendo que alguns destes também vão até o Campus durante o dia.

Além do questionário, os gráficos coletados mostraram os dados de forma que foi possível analisar e comparar com as respostas dos usuários. Desta maneira, nas Figuras 3 e 4 estão os dois gráficos da ferramenta Zabbix, sendo estes do dia 19 de junho de 2019 com os diferentes períodos ao longo de um dia.

Figura 3: Gráfico Zabbix - 07 às 18h

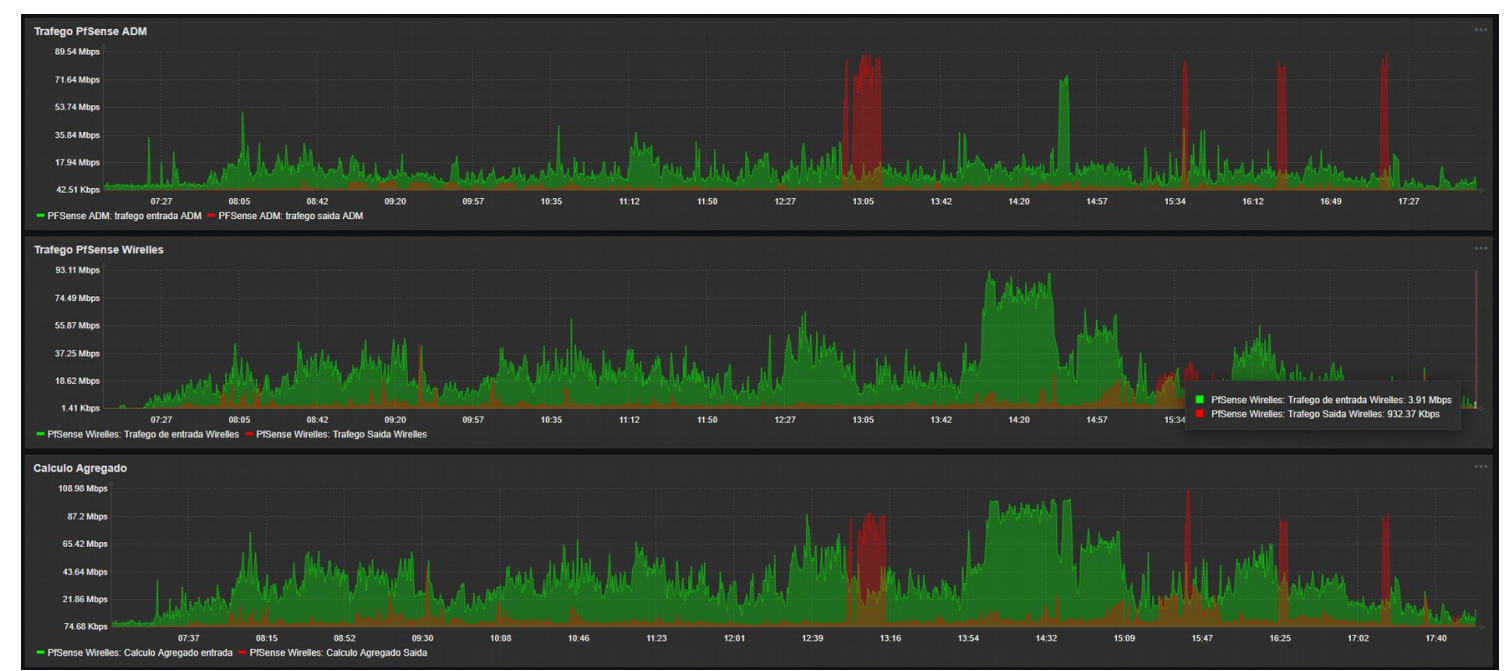

Fonte: IFC-CAS, 2019. 
Figura 4: Gráfico Zabbix - 18h às 23h

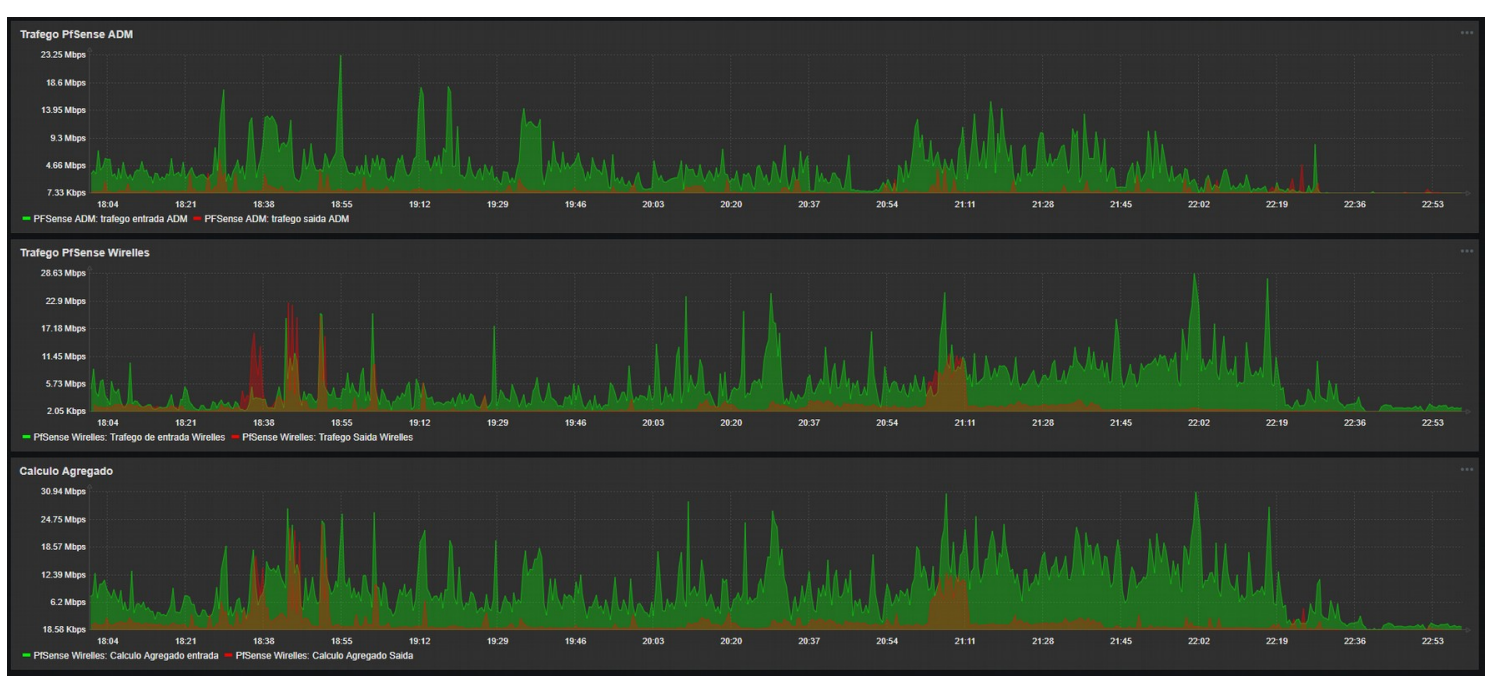

Fonte: IFC-CAS, 2019.

Comparando as respostas dos usuários com os gráficos, foi possível analisar e concluir que o horário do tráfego de dados para os usuários, o que possivelmente torna a maior lentidão na rede é das $19 \mathrm{~h}$ às $21 \mathrm{~h}$ e de acordo com os dados do Zabbix, isto acontece. Entretanto, é importante salientar que analisando os gráficos nota-se que a partir das 13h54 existe um pico maior em menos tempo (Figura 3). Ainda assim, tornase importante analisar a taxa de download e upload para definir melhores dados. Por fim, a unidade de transmissão de dados utilizada é o Mbps, que significa megabit por segundo.

\section{Considerações Finais}

Por meio deste trabalho um questionário foi desenvolvido e aplicado com os usuários da rede do Instituto Federal Catarinense - Campus Avançado Sombrio. As questões foram realizadas com o objetivo de conhecer a percepção do usuário em relação ao desempenho da rede e poder assim comparar com os dados que a ferramenta de monitoramento Zabbix possui.

Os questionamentos foram feitos com relação ao sinal do WiFi ser presente em todos os ambientes da instituição, se existe lentidão durante o uso e em qual período os usuários conseguem perceber esta lentidão. Entretanto, de acordo com as respostas negativas referentes as duas primeiras perguntas, como trabalhos futuros pretende-se explorar uma nova pesquisa para identificar onde o sinal do WiFi não está presente e quais os sites/aplicativos apresentam maior lentidão para os usuários. Por outro lado, pretende-se também utilizar os dados da ferramenta Zabbix com base na rede cabeada existente na Instituição e comparar com a rede $W i F i$, obtendo resultados e podendo analisar o desempenho das duas redes. Além disso, planeja-se analisar os equipamentos utilizados no IFC-CAS e avaliar também a percepção dos usuários dos turnos da manhã e da tarde.

Assim sendo, o objetivo deste trabalho foi alcançado, pois foi possível realizar a análise das respostas da percepção dos usuários e também dos gráficos de desempenho da rede. Desta maneira, a comparação entre ambos ocorreu fazendo com que respostas 
fossem obtidas e sugestões de trabalhos futuros fossem apresentadas.

\section{Referências}

Black, T. L. (2008). Comparação de ferramentas de gerenciamento de redes.

Forouzan, B. A. (2009). Comunicação de dados e redes de computadores. AMGH Editora.

De Franceschi, A. S. M., Barreto, J. M., \& Roisenberg, M. (2000). Desenvolvimento de Agentes Autônomos em gerência de Redes de Computadores. XVIII Simpósio Brasileiro de Telecomunicações, Gramado.

Franciscatto, R., Cristo, F. D., Perlin T. Redes de Computadores. Rede E-tec Brasil.

Gomes, J. C., \& Pizolato, R. P. (2018). Monitoramento da rede acadêmica do UNIFAFIBE e suas soluções.

Horst, A. S., dos Santos Pires, A., \& Déo, A. L. B. (2015). De A a Zabbix: Aprenda a monitorar e gerenciar aplicações e equipamentos de redes com o Zabbix. Novatec Editora.

Lima, J. D. R. (2014). Monitoramento de Redes com Zabbix: Monitore a saúde dos servidores e equipamentos de rede. Brasport.

Leonhardt, M. D. (2005). Doroty: um chatterbot para treinamento de profissionais atuantes no gerenciamento de redes de computadores.

ZABBIX. Zabbix: Site Oficial. [s.l]: Zabbix, 2019, de https://www.zabbix.com/. 